\title{
Diffusion Weighted Imaging in the Detection of Upper Urinary Tract Urothelial Tumors
}

\author{
Shuji Nishizawa, Shun Imai, Toshikazu Okaneya, Tsuyoshi Nakayama, Takayuki Kamigaito, \\ Tomonori Minagawa
}

Departments of Urology (SN, TO, TN, TK, TM) and Radiology (SI), Nagano Municipal Hospital, Nagano, Japan

\begin{abstract}
Purpose: Diffusion-weighted (DW) magnetic resonance imaging (MRI) provides information about the biophysical properties of tissues such as cell organization and density. DW imaging (DWI) is becoming important in the assessment of malignant tumors. The purpose of our study was to evaluate the capability and reliability of DWI in the evaluation of upper urinary tract urothelial tumors.

Materials and Methods: DWI was performed in seventeen patients with upper urinary tract urothelial tumor, previously diagnosed by either CT or retrograde pyelography. An histological evaluation was performed after surgical resection. Each MRI was carried out using a 1.5T superconductive magnet MRI system. DWI images were obtained with b value of 1000 $\mathrm{s} / \mathrm{mm}^{2}$ under normal breathing. The apparent diffusion coefficient (ADC) values were measured.

Results: In nine patients with renal pelvis tumors and seven patients with ureteral tumors, the lesions were shown as high-signal intensity in the corresponding region on DWI. In one patient with carcinoma in situ (CIS) of the ureter, the lesion was not depicted with DWI. The mean ADC value of the tumor was $1.125 \pm 0.217 \times 10^{-3} \mathrm{~mm}^{2} / \mathrm{s}$ and was significantly lower than those of the renal parenchyma $\left(1.984 \pm 0.238 \times 10^{-3} \mathrm{~mm}^{2} / \mathrm{s}, \mathrm{p}<0.01\right)$ and the urine $(2.941 \pm 0.315 \mathrm{x}$ $\left.10^{-3} \mathrm{~mm}^{2} / \mathrm{s}, \mathrm{p}<0.01\right)$.

Conclusions: In our study, the renal pelvic and ureteral tumors except CIS were shown clearly with DWI. Although further studies are required, DWI may take the place of invasive retrograde urography for detecting tumors of the upper urinary tract.
\end{abstract}

Key words: magnetic resonance imaging; transitional cell; neoplasm; renal pelvis; ureter

Int Braz J Urol. 2010; 36: 18-28

\section{INTRODUCTION}

Five percent of urothelial tumors occur from the ureter and renal pelvis or calyces, accounting for approximately $10 \%$ of upper urinary tract neoplasms (1). Upper urinary tract urothelial cancer is one of the most difficult lesions to be shown by imaging studies. Moreover, it is difficult to depict ureteral or renal pelvic small tumors. Conventionally, invasive radiography, such as retrograde pyelo-uretrography using cystoscopy, has been the imaging modality in detecting urothelial tumors.

Diffusion-weighted (DW) magnetic resonance imaging (MRI) is a technique used to show water molecular diffusion in vivo. It provides information about the biophysical properties of tissues such as cell organization and density, microstructure, and microcirculation (2). DW imaging (DWI) has been 
used in the field of neuroradiology. Recently, DWI has become increasingly important in the assessment of malignant tumors. Several authors have reported the usefulness of DWI in the detection of the abdominal and pelvic malignant lesion such as prostate cancer and colon cancer $(3,4)$. The purpose of our study was to evaluate the efficacy and reliability of DWI in the assessment of upper urinary tract urothelial tumors.

\section{MATERIALS AND METHODS}

\section{Patient Population}

This was a retrospective study performed at Nagano Municipal hospital. Between June 2003 and March 2007, seventeen patients with upper urinary tract urothelial tumor underwent MRI examination including DWI. All patients had upper tract urothe- lial tumor previously diagnosed either by computed tomography or by retrograde pyelography. Our Institutional Ethics Committee reviewed and approved the study protocol. Written informed consent was obtained from all patients.

The histological study was performed after surgical resection. The patients' characteristics are listed in Table-1.

Just before the examination, intramuscular or intravenous injection of $20 \mathrm{mg}$ of butyl scopolamine bromide was administered to all patients.

\section{Imaging Protocol}

Each MRI was performed using a $1.5 \mathrm{~T}$ superconductive magnet MRI system (Signa, Twin Speed Excite version 12.0, GE Medical Systems, Milwaukee, WI.) with maximum gradient amplitude of 40

Table 1 - Patients' characteristics.

\begin{tabular}{ccccccccc}
\hline $\begin{array}{c}\text { Case } \\
\text { N. }\end{array}$ & Age & Sex & Location & $\begin{array}{c}\text { Tumor } \\
\text { Size } \\
(\mathrm{mm})\end{array}$ & Classification & Grade & pT & $\begin{array}{c}\text { ADC Value } \\
\left(\mathrm{x} 10^{-3} \mathrm{~mm}^{2} / \mathrm{s}\right)\end{array}$ \\
\hline 1 & 73 & M & Renal pelvis & $\begin{array}{c}10, \\
\text { multiple }\end{array}$ & Papillary & G1-2 & pTa & 1.06 \\
2 & 61 & M & Renal pelvis & 20 & Papillary & G2 & pTa & 1.09 \\
3 & 58 & F & Renal pelvis & $40 \times 35$ & Papillary and infiltrating & G2 & pT3 & 0.884 \\
4 & 84 & M & Renal pelvis & $40 \times 45$ & Papillary and infiltrating & G2 & pT4 & 1.2 \\
5 & 72 & M & Renal pelvis & $20 \times 60$ & Papillary & G2 & pTa & 1.1 \\
6 & 56 & M & Renal pelvis & $45 \times 60$ & Papillary & G2 & pT1 & 1.33 \\
7 & 75 & M & Renal pelvis & $35 \times 50$ & Papillary & G1 2 & pT3 & 1.68 \\
8 & 78 & F & Renal pelvis & $20 \times 30$ & Papillary & G2-3 & pT3 & 1.26 \\
9 & 66 & M & Renal pelvis & $50 \times 35$ & Papillary and infiltrating & Sarcomatoid & pT3 & 1.11 \\
10 & 72 & M & Upper ureter & 30 & Papillary and infiltrating & G3 with SCC & pT3, pN0 & 1.17 \\
11 & 77 & M & Upper ureter & 10 & Papillary and infiltrating & G2 & pT1 & 0.927 \\
12 & 65 & M & Middle ureter & 10 & Infiltrating & G1-2 & pT1 & 1.02 \\
13 & 72 & M & Middle ureter & 8 & Infiltrating & G2 & pT2 + CIS & 0.944 \\
14 & 75 & M & Lower ureter & 8 & Infiltrating & G2-3 & pT2 +CIS & 1.00 \\
15 & 75 & M & Lower ureter & 10 & Infiltrating & G3 & pT3 & 0.752 \\
16 & 79 & M & Lower ureter & 15 & Papillary & G1 & pT3 & 1.22 \\
17 & 72 & M & Lower ureter & & CIS & G3 & CIS & \\
\hline
\end{tabular}

$A D C=$ apparent diffusion coefficient $; S C C=$ squamous cell carcinoma; $C I S=$ carcinoma in situ. 
Table 2 - Imaging parameters of diffusion-weighted imaging (DWI).

\begin{tabular}{lccc}
\hline Type of Scan & Non-breath-hold & Scan percentage & $100 \%$ \\
Sequence & SE-EPI & EPI factor & 64 \\
Mode & Single shot & ASSET factor & 2 \\
Coil & 8 Channel body & MPG & 3 axis \\
Slice orientation & Axial & b-factor & $1000 \mathrm{~s} / \mathrm{mm}^{2}$ \\
RT (repetition time) & $5000 \mathrm{~ms}$ & NEX (number of excitations) & 6 \\
ET (eco time) & $58.4 \mathrm{~ms}$ & Number of slices & $48 \times 2$ \\
Fat suppression & Water excitation & Slice thickness & $5 \mathrm{~mm}$ \\
FOV (field of view) & $350 \mathrm{~mm}$ & Slice gap & $0 \mathrm{~mm}$ \\
RFOV\% (receiver field of view) & $100 \%$ & Acquisition time & $240 \times 2 \mathrm{sec}$. \\
Matrix & 128 & & \\
\hline
\end{tabular}

$\mathrm{mT} / \mathrm{m}$ and a maximum slew rate of $150 \mathrm{mT} / \mathrm{m} / \mathrm{second}$, with an 8-channel-body array coil. DW images were obtained in the axial plane under normal breathing in addition to conventional T1/T2 weighted MR images without contrast-enhanced imaging. We obtained multiple axial thin slices DWI and reconstructed 3D images and maximum intensity projection (MIP) images. The imaging parameters used for DWI are listed in Table-2.

Typically, presence of the tumor was defined when high signal intensity appeared on DWI. Radiological diagnosis was performed by the same radiologist (S.I.).

The apparent diffusion coefficient (ADC) values of the tumor, the renal parenchyma and the urine in the bladder were calculated in a circular region of interest for quantitative analysis (Figure-1). Statistical analysis was performed by an un-paired t-test. Results are reported as mean \pm standard deviation. A p-value of less than 0.05 was considered statistically significant.

\section{RESULTS}

In nine patients with renal pelvic tumors, all lesions were shown as high-signal intensity in the renal pelvis or renal parenchyma on DWI (Figures-2 and 3), whereas conventional T1- and T2-weighted MRI was able to depict the lesion clearly in eight patients. In seven patients with a ureteral tumor, all tumors were depicted in the corresponding region (Figures-1 and 4). The smallest depicted tumor was approximately $8 \mathrm{~mm}$ in diameter. However, conventional MRI was able to depict ureteral tumor in five patients. In a patient (case 17) with carcinoma in situ (CIS) in the lower ureter and in two patients (cases 13 and 14) with associated CIS, DWI and conventional MRI failed to show the corresponding lesions. The sensitivity and positive predictive value (PPV) of DWI for detecting the tumor were $94.1 \%$ (16 of 17) and $100 \%$, respectively. The sensitivity and PPV of conventional MRI were $76.5 \%$ (13 of 17) and 100\%, respectively.

DWI showed a hyper-intense signal in several normal structures such as spleen, lymph node, spinal cord and mucus in the small intestine. Swollen lymph nodes in a patient (Figure-4, case 10) were shown as high signal intensity by DWI; however, the lymph nodes did not contain malignant cells on histopathological examination.

Histopathologically all tumors were diagnosed as a urothelial carcinoma in the surgical specimens. The cytological tests were negative in five patients with low-grade tumors.

The mean ADC value of the tumor was 1.125 $\pm 0.217 \times 10^{-3} \mathrm{~mm}^{2} / \mathrm{s}$, while the values of the renal parenchyma and the urine in the bladder were 1.984 $\pm 0.238 \times 10^{-3} \mathrm{~mm}^{2} / \mathrm{s}$ and $2.941 \pm 0.315 \times 10^{-3} \mathrm{~mm}^{2} / \mathrm{s}$, respectively. The mean $\mathrm{ADC}$ value of the urothelial tumor was significantly lower than those of the renal parenchyma and the urine $(\mathrm{p}<0.01$ and $\mathrm{p}<0.01)$. 

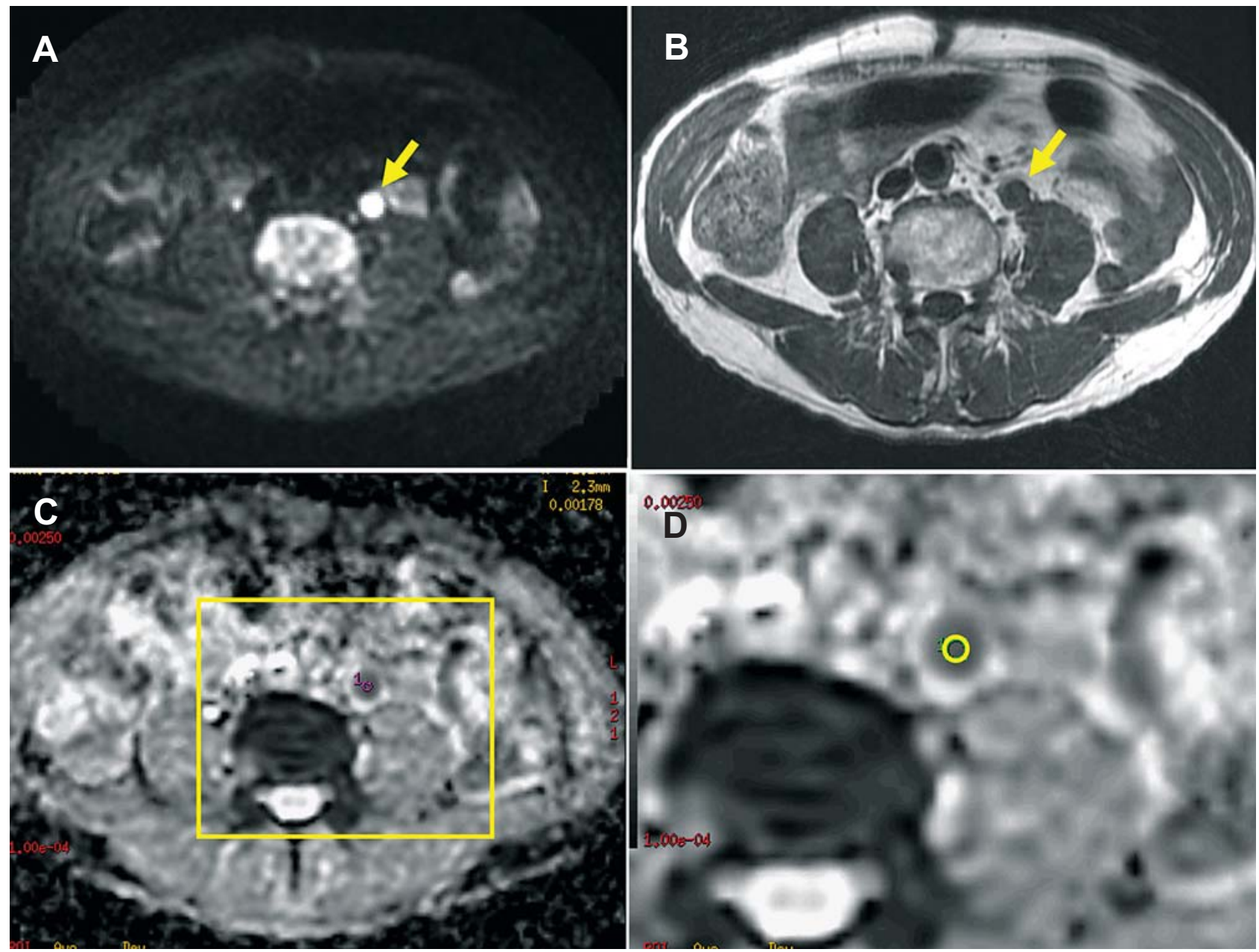

Figure 1-Case 11: A 77-year-old man with left ureteral tumor. A) On axial diffusion weighted image, the tumor (arrow) is depicted as high intensity. B) On axial T1-weighted image, the tumor shows iso-signal to psoas muscle. C) On apparent diffusion coefficient (ADC) map, the ADC value was measured in a circular region of interest within the tumor. D) Magnified view of the ADC map in $C$.

Regarding the tumor classification, the mean ADC value of infiltrating tumor was significantly lower than that of papillary tumor $\left(0.929 \pm 0.122 \times 10^{-3} \mathrm{~mm}^{2} / \mathrm{s}\right.$ and $\left.1.245 \pm 0.215 \times 10^{-3} \mathrm{~mm}^{2} / \mathrm{s}, \mathrm{p}<0.05\right)$.

\section{COMMENTS}

MRI has been infrequently used in the primary assessment of upper tract urothelial cancer, and the MRI characteristics of this tumor have not been well described. MRI imaging is independent of excretory function and shows multiplaner imaging, which permits direct image acquisition in the plane of tumor spread (5). Diffusion weighted imaging is an MRI technique and is the only imaging method that can evaluate the diffusion process in vivo. Diffusion is thermally induced motion of water molecules in biological tissues, which is called Brownian motion. The speed of diffusion of water molecules is different in the extracellular and intracellular component of the tissues. In the intracellular component, the diffusion is relatively slow because of the presence of cellular membranes (2). A malignant tumor often has a larger cell diameter and denser cellularity than normal tissue and the cell density may be indicative of tumor aggressiveness. Restriction of water diffusion is found to be a common feature of tumors (6). Apparent diffusion 


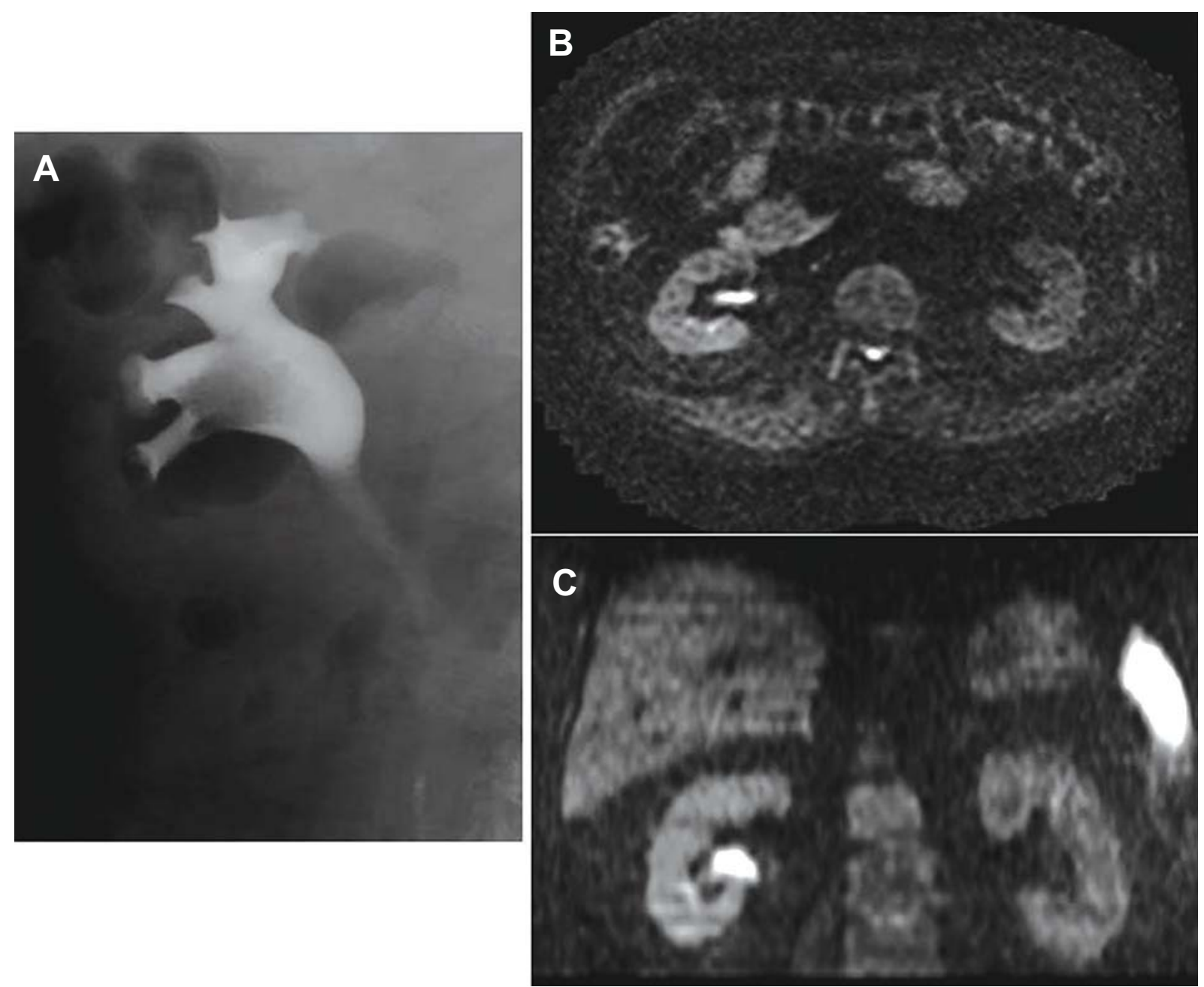

Figure 2-Case 2: A 61-year-old man with right renal pelvic tumor. A) Retrograde pyelography shows a filling defect in the renal pelvis. B) On axial diffusion weighted image (DWI) and C) Maximum intensity projection image obtained from axial DWIs, the tumor is depicted in the right renal pelvis as high intensity.

coefficient (ADC) values are quantitative expressions of diffusion characteristics of tissues, and ADC values are related to the proportion of extracellular and intracellular components. Since a malignant tumor often has a larger cell diameter and cellularly denser than normal tissue the ADC values of tumors may decrease (7). Therefore, DWI shows the tumor as high signal intensity as well. Takahara et al. showed the potential capability of DWI as a screening tool for malignancylike positron emission tomography. High-b-value DW-MRI images could be directly used for tumor detection because of the different cellular structures of healthy and neoplastic tissues. They reported a new DWI technique under normal breathing, which allows acquisition of more slices with multiple signal averaging, a higher signal-to-noise ratio, and high-quality MRI images (3). We used multi-excitation for data acquisition under normal breathing as well. Additional advantages of this technique are that it is completely non-invasive, and does not require exposure to ionizing radiation. Furthermore, adding this DWI to a routine MRI protocol requires only few minutes and does not cause patient discomfort. DWI does not require the administration of intravenous contrast material, which may cause allergic reaction or renal toxicity.

DWI is available for several malignancies including abdominal and pelvic lesions such as liver, colon, uterus and kidney and prostate cancer (8-12). 


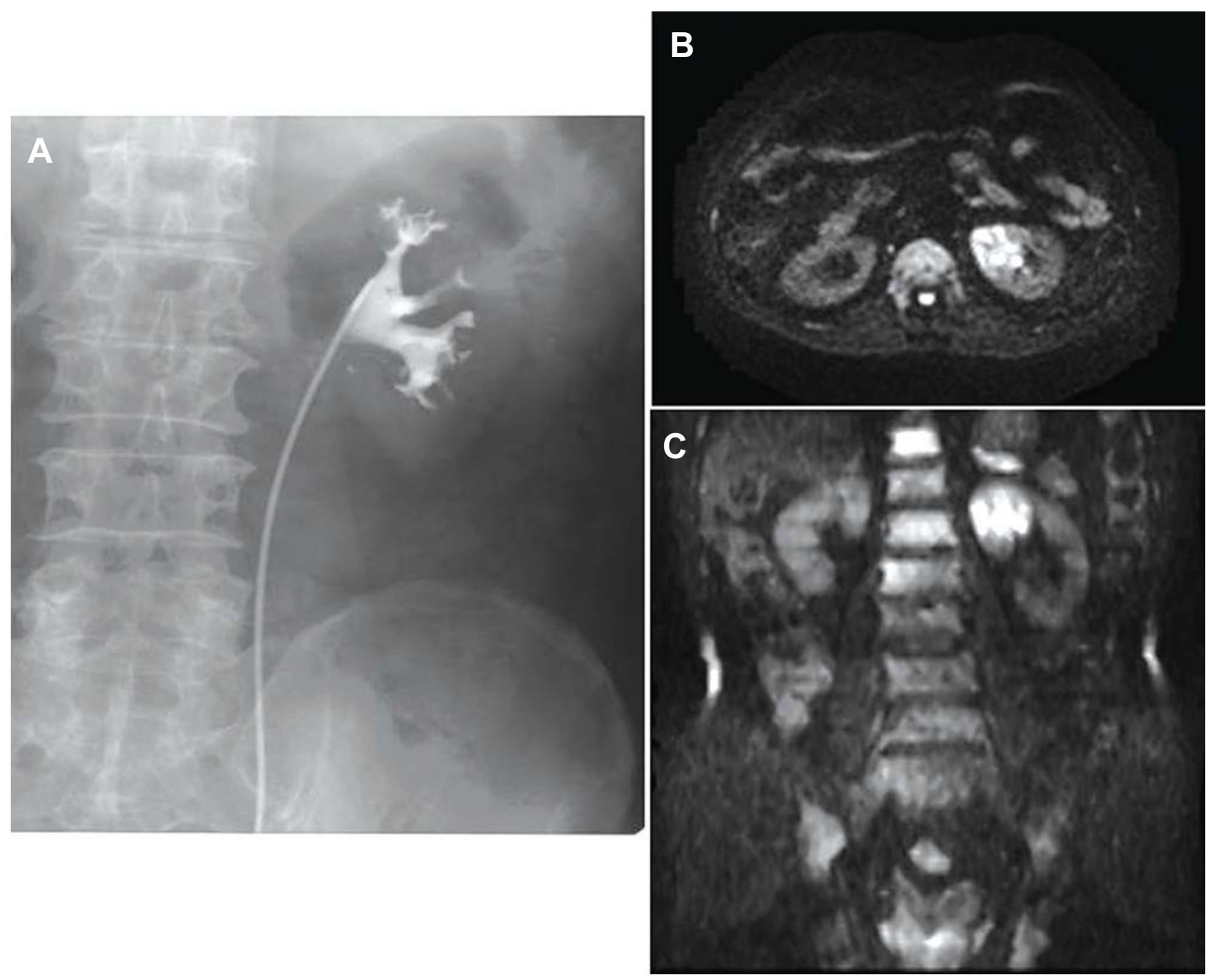

Figure 3 - Case 4: An 84-year-old man with left renal pelvic tumor. A) Retrograde pyelography shows small deformities in the upper calyx. B) On axial diffusion weighted image (DWI) and C) Maximum intensity projection image obtained from axial DWIs, the tumor is depicted in the parenchyma of upper part of the left kidney as high intensity.

Recently Yoshida et al. reported the application of DWI for a series of renal pelvic neoplasms (13), and Takeuchi et al. demonstrated the feasibility of this method for the detection of ureteral tumors (14), using DWI with diffusion gradient b-value of $800 \mathrm{~s} / \mathrm{mm}^{2}$. In these two reports, they demonstrated significantly lower ADC values of the tumors than of the surrounding tissues. Several authors have recently reported the feasibility of using DWI for the detection of a urinary bladder cancer (15-17).

In our study, the renal pelvic and ureteral tumors except CIS were clearly shown with DWI regardless of the tumor grade. The mean ADC value of the urothelial tumor was significantly lower than those of the renal tissue and the urine. DWI may detect the tumor of upper urinary tract more distinctly because urothelial tumors were surrounded by a fluid collection or urine. We were able to demonstrate that the mean $\mathrm{ADC}$ value of the infiltrating tumor was significantly lower than that of papillary tumor. This may depend on the difference of cellular density between the tumor types. Although Takeuchi et al. recently reported that the mean $\mathrm{ADC}$ value of G3 bladder cancer was significantly lower than that of $\mathrm{G} 1$ and G2 

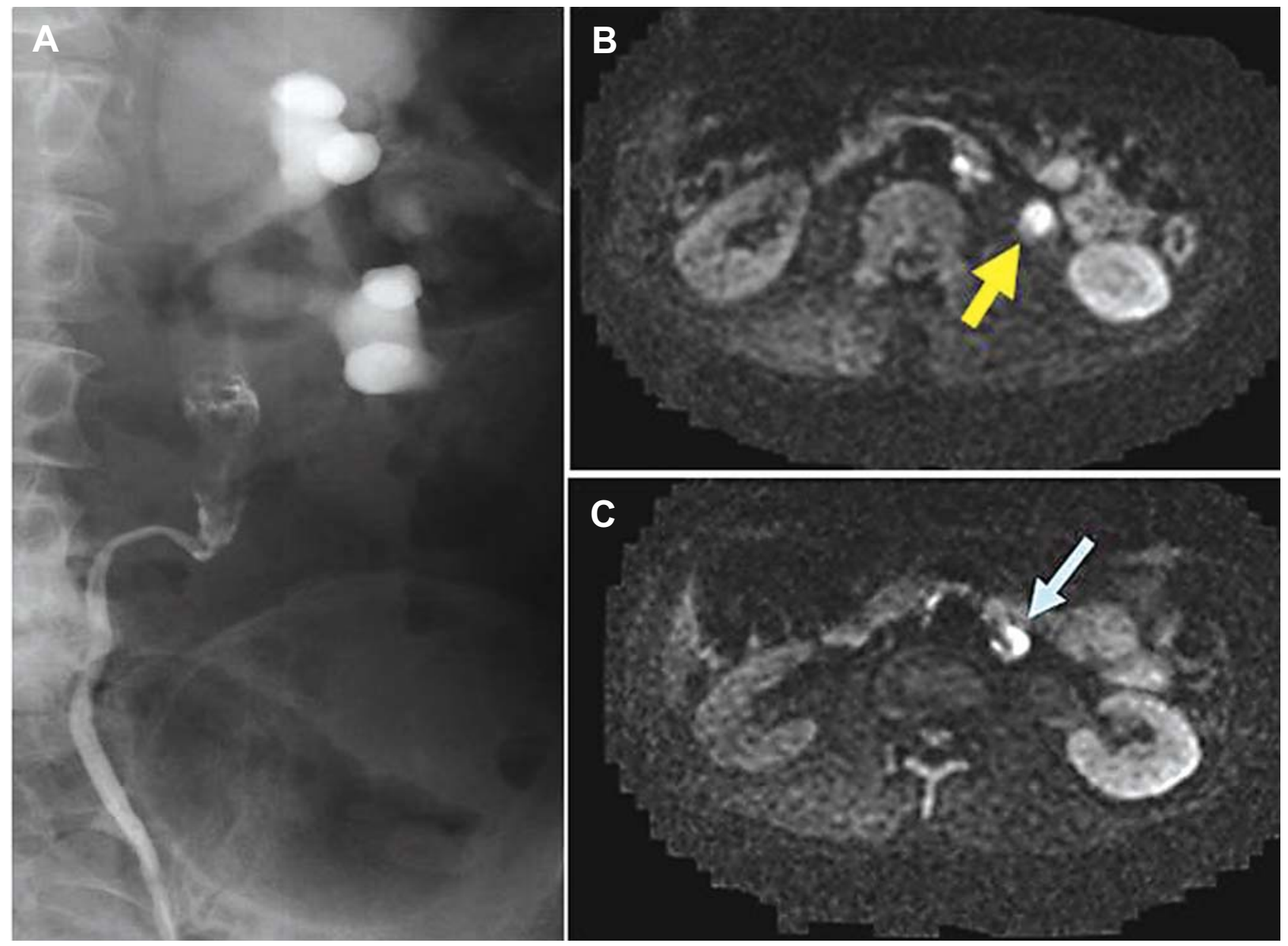

Figure 4-Case 10: A 72-year-old man with left ureteral tumor. A) Retrograde pyelography shows a filling defect in the upper ureter. B) On axial diffusion weighted image, the tumor is depicted in the upper ureter as high intensity. C) An enlarged para-aortic lymph node is shown as high signal intensity. Histopathologically, the lymph node was not a metastatic lesion.

tumors recently (17), we were not able to demonstrate the difference in ADC value between tumor grades. This may due to our small study population.

In our study, the smallest depicted tumor was approximately $8 \mathrm{~mm}$ in diameter. Yoshida et al. reported they were able to obtain high signal intensity of small renal pelvic tumors $(5 \mathrm{~mm}$ and $7 \mathrm{~mm}$ in diameter) on DWI, despite unclear conventional morphological MRI (14). DWI may provide the information about the characteristics of a small mass even if the mass is not clearly depicted by conventional MRI techniques. The CIS lesion in case 17 could not be depicted either in DWI or in conventional MRI. This was also not possible in the associated CIS lesions in the other patients. DWI may not be able to delineate the area of CIS at present because the lesion does not form a mass.

It might possibly be difficult to differentiate between malignant and benign tumors such as ureteral polyp by using conventional imaging studies and cytologic examination. Fujii et al. reported that the ADC values of uterine endometrial benign lesion including polyp and leiomyoma were significantly higher than that of the malignant lesion. They concluded that ADC measurement could provide useful information in differentiating malignant from benign uterine endometrial cavity lesions (18). Although we have no experience with the cases of benign urinary 
tract tumors such as polyp or endometriosis, DWI and $\mathrm{ADC}$ value may nevertheless provide the information about the property of the mass $(17,18)$.

An enlarged lymph node may be a false positive structure in the diagnosis of malignant tumor with DWI. Ichikawa et al. reported that most metastatic lymph nodes were detected because of their high signal intensity, in some patients healthy lymph nodes also showed similarly high signal intensities (4).

The standard work-up for the patient with hematuria consists of urinalysis and cytologic analysis, cystoscopy and excretory urography (5). Additional imaging is often required. The diagnosis of urothelial cancers is usually made based on the cytological analysis of urine specimens, which are collected on cystoscopy or retrograde pylography. These techniques are invasive and technically demanding. The preoperative cytologic studies were negative in five patients with low-grade tumors in our series. False negative cytologic results may occur in cases of low-grade lesions or in which the ureter is obstructed. MRI allows multiplanar images and MR urography can permit localization of ureteric obstruction. As previously mentioned, several reports have demonstrated the feasibility of DWI for the detecting upper and lower urinary tract tumor (1317). Although further studies are needed to prove the value of DWI for detecting upper urinary tract tumor and for differentiating malignant form benign urothelial tumor, MRI adding DWI may become the first choice for imaging studies, and DWI may replace invasive retrograde urography and imaging studies using intravenous injection of contrast medium.

This is the third reported study on the application of DWI for the detection of the upper urinary tract urothelial cancer. However, this study has several limitations. It is a retrospective study, assessing only 17 patients, and only one radiologist evaluated the images. Further studies with larger clinical settings are necessary.

\section{CONCLUSIONS}

In our study, the renal pelvic and ureteral cancers except CIS were shown clearly with DWI regardless of the tumor grade. We demonstrated that an infiltrating tumor had lower ADC values than that of papillary tumor. Although further studies with larger clinical settings are required, DWI may replace invasive retrograde urography or conventional imaging using intravenous injection of contrast medium in detecting tumors of the upper urinary tract.

\section{CONFLICT OF INTEREST}

None declared.

\section{REFERENCES}

1. Hall MC, Womack S, Sagalowsky AI, Carmody T, Erickstad MD, Roehrborn CG: Prognostic factors, recurrence, and survival in transitional cell carcinoma of the upper urinary tract: a 30-year experience in 252 patients. Urology. 1998; 52: 594-601.

2. Squillaci E, Manenti G, Cova M, Di Roma M, Miano $\mathrm{R}$, Palmieri G, et al.: Correlation of diffusion-weighted MR imaging with cellularity of renal tumours. Anticancer Res. 2004; 24: 4175-9.

3. Takahara T, Imai Y, Yamashita T, Yasuda S, Nasu S, Van Cauteren M: Diffusion weighted whole body imaging with background body signal suppression (DWIBS): technical improvement using free breathing, STIR and high resolution 3D display. Radiat Med. 2004; 22: 275-82.

4. Ichikawa T, Erturk SM, Motosugi U, Sou H, Iino H, Araki T, et al.: High-B-value diffusion-weighted MRI in colorectal cancer. AJR Am J Roentgenol. 2006; 187: 181-4.

5. Browne RF, Meehan CP, Colville J, Power R, Torreggiani WC: Transitional cell carcinoma of the upper urinary tract: spectrum of imaging findings. Radiographics. 2005; 25: 1609-27.

6. Sugahara T, Korogi Y, Kochi M, Ikushima I, Shigematu $\mathrm{Y}$, Hirai T, et al.: Usefulness of diffusion-weighted MRI with echo-planar technique in the evaluation of cellularity in gliomas. J Magn Reson Imaging. 1999; 9: 53-60.

7. Lyng H, Haraldseth O, Rofstad EK: Measurement of cell density and necrotic fraction in human melanoma xenografts by diffusion weighted magnetic resonance imaging. Magn Reson Med. 2000; 43: 828-36.

8. Namimoto T, Yamashita Y, Sumi S, Tang Y, Takahashi M: Focal liver masses: characterization with diffusion- 
weighted echo-planar MR imaging. Radiology. 1997; 204: 739-44.

9. Nasu K, Kuroki Y, Kuroki S, Murakami K, Nawano S, Moriyama N: Diffusion-weighted single shot echo planar imaging of colorectal cancer using a sensitivity-encoding technique. Jpn J Clin Oncol. 2004; 34 : 620-6.

10. Shimofusa R, Fujimoto H, Akamata H, Motoori K, Yamamoto S, Ueda T, et al.: Diffusion-weighted imaging of prostate cancer. J Comput Assist Tomogr. 2005; 29: 149-53.

11. Charles-Edwards EM, deSouza NM: Diffusionweighted magnetic resonance imaging and its application to cancer. Cancer Imaging. 2006; 6: 135-43.

12. Squillaci E, Manenti G, Di Stefano F, Miano R, Strigari L, Simonetti G: Diffusion-weighted MR imaging in the evaluation of renal tumours. J Exp Clin Cancer Res. 2004; 23: 39-45.

13. Yoshida S, Masuda H, Ishii C, Saito K, Kawakami S, Kihara K: Initial experience of functional imaging of upper urinary tract neoplasm by diffusion-weighted magnetic resonance imaging. Int J Urol. 2008; 15: 140-3.
14. Takeuchi M, Matsuzaki K, Kubo H, Nishitani H: Diffusion-weighted magnetic resonance imaging of urinary epithelial cancer with upper urinary tract obstruction: preliminary results. Acta Radiol. 2008; 49: 1195-9.

15. Matsuki M, Inada Y, Tatsugami F, Tanikake M, Narabayashi I, Katsuoka Y: Diffusion-weighted MR imaging for urinary bladder carcinoma: initial results. Eur Radiol. 2007; 17: 201-4.

16. El-Assmy A, Abou-El-Ghar ME, Refaie HF, El-Diasty T: Diffusion-weighted MR imaging in diagnosis of superficial and invasive urinary bladder carcinoma: a preliminary prospective study. ScientificWorldJournal. 2008; 8: 364-70.

17. Takeuchi M, Sasaki S, Ito M, Okada S, Takahashi S, Kawai T, et al.: Urinary bladder cancer: diffusionweighted MR imaging--accuracy for diagnosing $\mathrm{T}$ stage and estimating histologic grade. Radiology. 2009; 251: 112-21.

18. Fujii S, Matsusue E, Kigawa J, Sato S, Kanasaki Y, Nakanishi J, et al.: Diagnostic accuracy of the apparent diffusion coefficient in differentiating benign from malignant uterine endometrial cavity lesions: initial results. Eur Radiol. 2008; 18: 384-9.
Accepted after revision: August 11, 2009

\section{Correspondence address:}

Dr. Shuji Nishizawa

Nagano Municipal Hospital

1333-1 Tomitake, Nagano, 381-8551, Japan

Fax: + 8126 295-1145

E-mail: sxnishizawa@hospital.nagano.nagano.jp

\section{EDITORIAL COMMENT}

Urothelial carcinoma of the upper urinary tract accounts for $5 \%$ of all urothelial carcinomas and $10 \%$ of all renal tumors. Patients with a history of urothelial carcinoma are at high risk of developing synchronous or metachronous urothelial carcinoma. This known multifocality requires thorough examination of the entire urinary tract in high-risk patients
(1). The diagnosis is difficult by conventional morphological imaging, since urothelial carcinomas may be overlooked with suboptimal examinations and even though the examination is sufficient, small lesions may not be detected due to volume averaging (2).

Excretory urography or computed tomography (CT) was used to evaluate high-risk patients (3). 
Multiphasic CT urography offers superior detection of calculi, urothelial tumor, and parenchymal tumor over excretory urography and allows accurate staging of detected lesions at the same examination. However, when the patient has a contraindication to iodinated contrast material, retrograde pyelography or MRI is often used to image the upper urinary tract (4). MR imaging, including MR angiography and MR urography, offers comparable evaluation in patients who cannot tolerate iodinated contrast material and in whom multiplanar, vascular, and collecting system imaging is required $(3,4)$.

Recently a new MRI technique, called diffusion-weighted MR imaging (DW-MRI) has been applied in various abdominal diseases, especially in detecting tumors without the need for contrast administration (5). Different from conventional anatomical MR imaging DW-MRI provide functional information (5). By studying molecular diffusion, the ultrastructural characteristics of tissue can be studied in vivo through sampling water molecules and by exploiting the natural sensitivity of MRI to the motion (2).

The research of Nishizawa et al. has shown the superiority of DW-MRI in detecting urothelial tumors with a very high accuracy, demonstrated with excellent images. Also in a study by Yoshida et al. two cases of highly suspected upper urinary tract neoplasm had been detected clearly on DW-MR images, despite unclear conventional morphological MR images (2).

Malignant masses are easily discernible against suppressed background signal with visual assessment of DW-MR images (PET like images).
This method may be obtained after a routine abdominal MR imaging protocol approximately in 3 to 5 minutes without an additional cost. The additional benefit of DW-MRI is the ability to determine quantitative indices, which may be important in the assessment of tumor cellularity, and disease response to treatment methods and follow-up $(2,5)$.

Further investigations will probably increase the use of functional imaging methods and especially DW-MRI in genitourinary diseases.

\section{REFERENCES}

1. Zhang J, Lefkowitz RA, Bach A: Imaging of kidney cancer. Radiol Clin North Am. 2007; 45: 119-47.

2. Yoshida S, Masuda H, Ishii C, Saito K, Kawakami $\mathrm{S}$, Kihara K: Initial experience of functional imaging of upper urinary tract neoplasm by diffusion-weighted magnetic resonance imaging. Int J Urol. 2008; 15: 140-3.

3. Takahashi N, Kawashima A, Glockner JF, Hartman RP, Leibovich BC, Brau AC, ET AL.: Small $(<2-\mathrm{cm})$ upper-tract urothelial carcinoma: evaluation with gadolinium-enhanced three-dimensional spoiled gradient-recalled echo MR urography. Radiology. 2008; 247: 451-7.

4. Browne RF, Meehan CP, Colville J, Power R, Torreggiani WC: Transitional cell carcinoma of the upper urinary tract: spectrum of imaging findings. Radiographics. 2005; 25: 1609-27.

5. Manenti G, Di Roma M, Mancino S, Bartolucci DA, Palmieri G, Mastrangeli R, et al.: Malignant renal neoplasms: correlation between $\mathrm{ADC}$ values and cellularity in diffusion weighted magnetic resonance imaging at 3 T. Radiol Med. 2008; 113: 199-213.

Dr. Ozgur Kilickesmez

Department of Radiology Yeditepe University Hospital Istanbul, Turkey E-mail:okilickesmez@yahoo.com 


\section{EDITORIAL COMMENT}

A combination of morphological imaging modalities using iodinated radiocontrast media, such as excretory urography and computed tomography with urinary cytologic examination, has been used for diagnosing upper urinary tract cancer (UUTC). However, these media may cause renal insufficiency. Contrast-induced nephropathy due to iodinated radiocontrast media is currently the third most common cause of acute renal failure in hospitalized patients (1). Diffusion-weighted magnetic resonance imaging (DWI) is a functional imaging technique with no contrast agent and is applicable to patients with allergies against contrast agents or existing renal insufficiency. Furthermore, the addition of DWI to a routine MRI examination can be readily adopted for most current clinical MRI scanners with only a few minutes and no additional equipment.

DWI has been reported to be a useful technique to detect UUTC in a noninvasive manner because of clear contrast between high signal intensity of UUTC and well-restrained signal intensity of the surrounding tissue (2). This study has confirmed the above points. The location (pelvis or ureter) and the size of the UUTC seem to have little impact on diagnostic potentiality because of good contrast between the tumor and the surrounding tissues, even if the tumor burden was small $(8 \mathrm{~mm}$ in this paper, $5 \mathrm{~mm}$ in our experience). The impact in the degree of diffusion within the tumor on the estimation of grade or depth has recently been shown on DWI in some malignancies, including bladder cancer (3). In this study, ADC values of the infiltrating tumor were significantly lower than that of papillary tumor. However, overlap among ADC values between them seems to exist. Also, overlap among ADC values for tumor, renal parenchyma and collecting system exists. Further investigations should be performed to clarify the clinical importance of ADC values in evaluating tumor aggressiveness.

The current study also showed the limitation of DWI technique in assessing the UUTC. Depicting carcinoma in situ (CIS) lesions is also challenging in DWI, as well as in other conventional imaging modalities. The DWI contrast reflects molecular diffusion, not the presence of existing cancer cells. Therefore, it remains challenging to distinguish a malignant disease from nonmalignant lesions, such as benign neoplasms, hematomas, abscesses and inflammatory condition.

We have to keep in mind that we could not gain anatomical information from DWI. Addition of DWI to anatomical imaging increases the accuracy of MRI to UUTC.

\section{REFERENCES}

1. Solomon R: Contrast-medium-induced acute renal failure. Kidney Int. 1998; 53: 230-42. Erratum in: Kidney Int 1998; 53: 818. published erratum of serious dosage error appears in Kidney Int 1998; 53: 1109.

2. Yoshida S, Masuda H, Ishii C, Saito K, Kawakami S, Kihara K: Initial experience of functional imaging of upper urinary tract neoplasm by diffusion-weighted magnetic resonance imaging. Int J Urol. 2008; 15: 140-3.

3. Abou-El-Ghar ME, El-Assmy A, Refaie HF, El-Diasty T: Bladder cancer: diagnosis with diffusion-weighted MR imaging in patients with gross hematuria. Radiology. 2009; 251: 415-21.

Dr. Hitoshi Masuda Department of Urology Tokyo Medical and Dental University Graduate School Tokyo, Japan E-mail:hi-masu.uro@tmd.ac.jp 